\title{
Validade da Rotina de Realização do Eletrocardiograma na Avaliação Pré-Operatória de Idosos *
}

\section{The Validity of the Electrocardiogram Accomplishment in the Elderly Surgical Patient Preoperative Evaluation}

Flávia Salles de Souza ${ }^{1}$; José Ricardo Pinotti Pedro ${ }^{1}$; Joaquim Edson Vieira, TSA ${ }^{2}$; Arthur Vitor Rosenti Segurado ${ }^{1}$; Marcos Paulo Ferreira Botelho ${ }^{3}$; Ligia Andrade da Silva Telles Mathias, TSA ${ }^{4}$

\section{RESUMO}

Souza FS, Pedro JRP, Vieira JE, Segurado AVR, Botelho MPF, Mathias LAST - Validade da Rotina de Realização do Eletrocardiograma na Avaliação Pré-Operatória de Idosos

JUSTIFICATIVA E OBJETIVOS: Com os avanços médicos e o aumento da expectativa de vida, a população de idosos submetidos a procedimentos cirúrgicos vem aumentando. $O$ paciente idoso tem maior morbiletalidade cardíaca peri-operatória do que o paciente jovem, mesmo quando assintomático. 0 eletrocardiograma (ECG) tem sido solicitado no período pré-operatório na tentativa de reduzir a morbiletalidade intra-operatória, porém sua eficácia é controversa. Não há consenso na literatura a respeito da indicação do ECG em pacientes idosos no período pré-operatório, o que motivou a realização deste estudo. O objetivo foi verificar retrospectivamente a validade da rotina de realização do ECG no período pré-operatório numa população de pacientes idosos cirúrgicos.

MÉTODO: Análise retrospectiva de prontuários de pacientes a partir de 60 anos, submetidos a cirurgias diversas, durante o período de 6 meses. Os dados foram coletados para análise descritiva da população estudada; avaliação da incidência e dos tipos mais freqüentes de anormalidade dos ECG; análise comparativa dos pacientes com ECG normal e alterado em relação às diversas variáveis - idade, faixa etária, estado físico, presença de doença cardiovascular (DCV), complicações intra (CIO) e pós-operatórias (CPO).Também foram avaliados os pacientes sem DCV e aqueles com estado físico ASA I em relação às $\mathrm{ClO} e$ $C P O$, segundo os tipos de alterações do ECG e faixa etária.

\footnotetext{
* Recebido do (Received from) Centro de Ensino e Treinamento/SBA, Serviço de Anestesia da Irmandade Santa Casa de Misericórdia de São Paulo (ISCMSP), São Paulo, SP

1. Médico Assistente, Serviço de Anestesiologia da Irmandade Santa Casa de Misericórdia de São Paulo

2. Coordenador do Centro para Desenvolvimento da Educação Médica CEDEM da Faculdade de Medicina da Universidade de São Paulo

3. Graduando do $6^{\circ}$ ano da Faculdade de Ciências Médicas da Santa Casa de São Paulo

4. Diretora do Serviço e Disciplina de Anestesia, Irmandade Santa Casa de Misericórdia de São Paulo e Faculdade de Ciências Médicas da Santa Casa de São Paulo; Responsável pelo Centro de Ensino e Treinamento, CET/SBA, ISCMSP
}

Apresentado (Submitted) em 25 de maio de 2004

Aceito (Accepted) para publicação em 15 de outubro de 2004

Endereço para correspondência (Correspondence to)

Dra. Ligia Andrade da Silva Telles Mathias

Alameda Campinas, 139/41

01404-000 São Paulo, SP

E-mail: rtimao@uol.com.br

(C) Sociedade Brasileira de Anestesiologia, 2005
RESULTADOS: Foram analisados os prontuários de 481 pacientes, dos quais 287 continham ECG e destes, 88,8\% apresentavam anormalidades, sendo a mais freqüente, a alteração da repolarização ventricular. Não foi observada influência das variáveis estudadas sobre a incidência de ECG alterados. A incidência de alterações do ECG aumentou com o avanço da idade em todos pacientes estudados. Com o avanço da idade também ocorreu aumento da incidência de pacientes com ECG alterados associados com complicações intra-operatórias. As anormalidades eletrocardiográficas foram relevantes em relação à incidência de complicações intra-operatórias em todos os grupos estudados, principalmente as alterações secundárias à isquemia.

CONCLUSÕES: Este estudo mostrou que, para a população cirúrgica idosa estudada, é válida a rotina de realização do eletrocardiograma como parte da avaliação pré-operatória.

Unitermos: ANESTESIA, Idoso; AVALIAÇÃO PRÉOPERATÓRIA; EXAMES PRÉ-OPERATÓRIOS: eletrocardiografia

\section{SUMMARY}

Souza FS, Pedro JRP, Vieira JE, Segurado AVR, Botelho MPF, Mathias LAST - The Validity of the Electrocardiogram Accomplishment in the Elderly Surgical Patient Preoperative Evaluation

BACKGROUND AND OBJECTIVES: Because of medical progress, life expectancy has been prolonged and the elderly population submitted to surgical procedures has been growing. Besides age-related cardiovascular changes, the prevalence of diseases, such as systemic hypertension, affect cardiovascular reserve and increase morbidity, mortality and perioperative outcomes. Electrocardiogram is useful in diagnosing previous myocardial infarction and arrhythmias. In the preoperative evaluation of these patients, ECG usefulness is a controversial subject. This study aimed at retrospectively evaluating the validity of preoperative ECG in elderly patients.

METHODS: Retropective analysis of 481 patient records over 60 years old, submitted to different surgical procedures. Data were collected for description of the studied group; evaluation of most frequent electrocardiography abnormalities; comparative analysis between patients with normal and abnormal ECG related to age, ASA physical status, presence of cardiac disease intra and postoperative complications. Patients without cardiovascular disease (CVD) and physical status ASA I were separately analyzed. RESULTS: There were 481 patients, 287 of them with preoperative ECG and from those, 88, $8 \%$ had abnormalities, being the most frequent ventricular repolarization changes. There was not influence of the parameters on the frequency of abnormal ECG. The abnormalitees of the ECG, especially those secondary to ischemia were related with intraoperative complications in all study groups.

CONCLUSIONS: This study has shown that electrocardiogram is a valid routine for preoperative evaluation of elderly patients.

Key Words: ANESTHESIA, Elderly; PREOPERATIVE EVALUATION; PREOPERATIVE TESTS: electrocardiography 


\section{INTRODUÇÃO}

$\mathrm{N}$ os últimos 100 anos tem aumentado a expectativa de vida da população e o progresso da Medicina parece ser o fator que mais contribuiu para que isso ocorresse. Mais da metade dos indivíduos idosos será submetida a pelo menos uma intervenção cirúrgica até o fim de suas vidas e a comunidade anestesiológica deve se preparar para avaliar e atender este crescente número de pacientes ${ }^{1}$.

Em relação ao sistema cardiovascular, o avanço da idade está ligado a uma grande variedade de alterações estruturais e funcionais no coração e nos vasos. Além das alterações fisiológicas da idade, há aumento da prevalência de doenças cardiovasculares subjacentes (hipertensão arterial sistêmica, aterosclerose coronariana, infarto agudo do miocárdio, insuficiência cardíaca congestiva) que limitam ainda mais a função deste sistema ${ }^{2,3}$.

O objetivo principal da avaliação pré-operatória e pré-anestésica é sempre, independente da idade do paciente, a redução da morbiletalidade peri-operatória. Nesse sentido, atualmente, os exames de laboratório têm sido indicados prioritariamente, de acordo com dados da história e/ou do exame físico ${ }^{4}$.

No caso do eletrocardiograma (ECG), este tem sido solicitado de rotina no período pré-operatório na tentativa de se identificar doenças cardiovasculares e reduzir a morbiletalidade intra-operatória, porém, é controversa a sua influência nas condutas no pré e intra-operatório, para prevenir complicações cardiovasculares durante a cirurgia ${ }^{5-7}$.

Vários autores descreveram aumento da incidência de alterações eletrocardiográficas com a idade ${ }^{8-11}$.

É conhecido o fato de que isquemia miocárdica silenciosa (IMS) ocorre com maior freqüência nos idosos ${ }^{12}$ e que o infarto recente é forte preditor de risco cardiovascular peri-operatório aumentado ${ }^{13}$.

No entanto, ainda persiste a divergência na literatura com relação à acurácia do ECG pré-operatório como preditor de complicações cardiovasculares peri-operatórias no paciente idoso e, conseqüentemente, quanto à sua indicação como exame de rotina para este grupo de pacientes ${ }^{10-12,14-16}$.

Assim, decidiu-se realizar este estudo com o objetivo de avaliar a validade da indicação do ECG no período pré-operatório numa população determinada de pacientes idosos cirúrgicos.

\section{MÉTODO}

Após ter sido aprovado pelo Comitê de Ética em Pesquisa do Hospital Central da Irmandade da Santa Casa de Misericórdia de São Paulo, este estudo retrospectivo consistiu no levantamento, durante o período de primeiro de abril a trinta de setembro de 2000, de prontuários de pacientes idosos (idade a partir de 60 anos), submetidos a procedimentos cirúrgicos diversos sob anestesia, com eletrocardiogramas realizados durante a internação no período pré-operatório ou em regime ambulatorial até 6 meses antes da internação, com laudo emitido por cardiologista ou clínico geral da instituição.
Os dados obtidos dos prontuários foram: sexo; idade; estado físico (ASA); presença de sinais ou sintomas de doença cardiovascular e outras doenças associadas; eletrocardiograma; tipo de cirurgia e técnica anestésica; complicações hemodinâmicas intra-operatórias não relacionadas a efeitos adversos do ato cirúrgico, (redução/aumento da freqüência cardíaca ou pressão arterial maior que $20 \%$ dos valores basais e disritmias cardíacas) e complicações pós-operatórias. Os dados foram avaliados de acordo com a seguinte seqüência:

1. Verificação da incidência de ECG realizados nas condições estabelecidas neste estudo;

2. Verificação da incidência de ECG normais e alterados em relação às variáveis: idade; distribuição por faixa etária (60 a 69; 70 a 79 e $\geq 80$ anos), estado físico, presença de doença cardiovascular, complicações intra e pós-operatórias. Verificação da incidência de ECG alterados associados com complicações intra e pós-operatórias, de acordo com a distribuição por faixa etária, verificação dos tipos de alterações eletrocardiográficas e a incidência de complicações intra e pós-operatórias relacionadas às alterações observadas;

3. Análise dos pacientes sem doença cardiovascular e dos pacientes ASA I para: verificação da incidência de ECG alterados de acordo com a distribuição por faixa etária e da incidência de complicações intra e pós-operatórias associadas; verificação dos tipos de alterações eletrocardiográficas e a incidência de complicações intra e pós-operatórias relacionadas às alterações observadas.

Foi realizada análise descritiva dos resultados. Na comparação entre os resultados referentes à idade, foi utilizado o teste $t$ de Student não pareado. Na comparação entre os resultados referentes às outras variáveis, foi utilizado o teste do Qui-quadrado $\left(\chi^{2}\right)$. Foi considerada diferença estatisticamente significativa quando $p<0,05$. Os testes utilizados fazem parte do conjunto estatístico Sigma Stat for Windows, version 2.03, SPSS Inc.

\section{RESULTADOS}

Foram analisados 481 prontuários dos quais 194 foram excluídos por se tratarem de pacientes submetidos a atos anestésico-cirúrgicos sem eletrocardiograma, ou com ECG realizado fora do protocolo deste estudo. O número total de pacientes incluídos na pesquisa, portanto, foi de 287 e destes, 255 apresentavam laudos com alterações $(88,85 \%)$.

As idades médias (desvios-padrão) dos pacientes com ECG normal e ECG alterado foram, respectivamente, $74,2(7,1)$ e $73,5(8,2)$ anos, não havendo diferença estatística entre os dois grupos (teste $t$ não pareado - $p=0,643$ ). Também não foi observada diferença significativa em relação à distribuição dos pacientes quanto ao sexo, havendo maioria do sexo masculino em ambos os grupos $\left(\chi^{2}-p=0,544\right)$. 
A distribuição dos pacientes com ECG normal e alterado de acordo com o estado físico (ASA) não mostrou diferença significativa entre os dois grupos $\left(\chi^{2}\right)$, havendo maioria de pacientes com ECG alterado, independente do estado físico (Tabela I).

Tabela I - Distribuição dos Pacientes com Eletrocardiograma Normal e Alterado em Relação à Classificação do Estado Físico e Resultado da Análise Estatística $\left(\chi^{2}\right)$

\begin{tabular}{lccc}
\hline Estado Físico & ECG Normal & ECG Alterado & Total \\
\hline ASA I & $6(9,8 \%)$ & $55(90,2 \%)$ & 61 \\
ASA II & $22(11,9 \%)$ & $163(88,1 \%)$ & 185 \\
ASA III & $4(10,5 \%)$ & $34(89,5 \%)$ & 38 \\
ASA IV & $0(0 \%)$ & $3(100 \%)$ & 3 \\
\hline Total & 32 & 255 & 287 \\
\hline
\end{tabular}

$\chi^{2}=$ teste do Qui-quadrado $-p=0,722$

Quando os pacientes com ECG normal e alterado foram comparados em relação à presença de doença cardiovascular sintomática (DCV) e de complicações hemodinâmicas intra-operatórias $(\mathrm{ClO})$ e pós-operatórias ( $\mathrm{CPO})$, não foi observada diferença estatisticamente significativa entre os dois grupos, ou seja, a proporção de pacientes com e sem DCV; com e sem ClO; com e sem CPO foi similar em ambos os grupos com ECG normal e alterado (Tabela II).

Tabela II - Distribuição dos Pacientes com ECG Normal e Alterado em Relação à Presença de Doença Cardiovascular, Complicações Intra e Pós-Operatórias e Resultado da Análise Estatística $\left(\chi^{2}\right)$

\begin{tabular}{|c|c|c|c|c|}
\hline & & $\begin{array}{c}\text { ECG } \\
\text { Normal } n(\%)\end{array}$ & $\begin{array}{c}\text { ECG } \\
\text { Alterado } \mathrm{n}(\%)\end{array}$ & $p\left(\chi^{2}\right)$ \\
\hline \multirow[t]{2}{*}{$\begin{array}{l}\text { Doença } \\
\text { cardiovascular }\end{array}$} & $\begin{array}{l}\operatorname{sim} \\
(n=192)\end{array}$ & $\begin{array}{c}19 \\
(59,4)\end{array}$ & $\begin{array}{c}173 \\
(67,8)\end{array}$ & 0,255 \\
\hline & $\begin{array}{c}\text { não } \\
(n=95)\end{array}$ & $\begin{array}{c}13 \\
(40,6)\end{array}$ & $\begin{array}{c}82 \\
(32,2)\end{array}$ & \\
\hline \multirow[t]{2}{*}{$\begin{array}{l}\text { Complicações } \\
\text { intra-operatórias }\end{array}$} & $\operatorname{sim}_{(n=89)}$ & $\begin{array}{c}7 \\
(21,9)\end{array}$ & $\begin{array}{c}82 \\
(32,1)\end{array}$ & 0,155 \\
\hline & $\begin{array}{c}\text { não } \\
(n=198)\end{array}$ & $\begin{array}{c}25 \\
(78,1)\end{array}$ & $\begin{array}{c}173 \\
(67,9)\end{array}$ & \\
\hline \multirow[t]{2}{*}{$\begin{array}{l}\text { Complicações } \\
\text { pós-operatórias }\end{array}$} & $\operatorname{sim}_{(n=29)}$ & $\begin{array}{c}1 \\
(3,1)\end{array}$ & $\begin{array}{l}28 \\
(11)\end{array}$ & 0,338 \\
\hline & $\begin{array}{c}\text { não } \\
(\mathrm{n}=258)\end{array}$ & $\begin{array}{c}31 \\
(96,9)\end{array}$ & $\begin{array}{l}227 \\
(89)\end{array}$ & \\
\hline
\end{tabular}

A figura 1 mostra a distribuição por faixa etária do número total de pacientes com ECG realizados, alterados e normais. Houve diferença estatística na comparação, por faixa etária, do número de ECG normale ECG alterado $\left(\chi^{2} ; p=0,0006\right)$.

$\mathrm{Na}$ tabela III pode-se verificar os resultados relativos à distribuição por faixa etária do número total de pacientes com ECG alterado com e sem CIO e CPO e o resultado da análise estatística $\left(\chi^{2}\right)$, observando-se que houve aumento significativo das $\mathrm{ClO}$ com o aumento da idade $(p=0,0146)$, o que não foi verificado em relação às CPO $(p=0,0799)$.
Tabela III - Distribuição por Faixa Etária do Número Total de Pacientes com ECG Alterados e do Número de Pacientes com ECG Alterados com/sem CIO e CPO e Resultado da Análise Estatística $\left(\chi^{2}\right)$

\begin{tabular}{|c|c|c|c|}
\hline \multirow{2}{*}{$\begin{array}{l}\text { Faixa Etária } \\
\text { (anos) }\end{array}$} & \multirow[b]{2}{*}{ Total } & \multicolumn{2}{|c|}{ Total de Pacientes } \\
\hline & & Com/Sem ClO & Com/Sem CPO \\
\hline 60 a 69 & 79 & $19 / 60$ & $13 / 66$ \\
\hline 70 a 79 & 122 & $39 / 83$ & $11 / 111$ \\
\hline$>79$ & 54 & $24 / 30$ & $4 / 50$ \\
\hline $\begin{array}{l}\text { Total } \\
\mathrm{p}\end{array}$ & 255 & $\begin{array}{l}82 / 173 \\
0,0146\end{array}$ & $\begin{array}{l}28 / 227 \\
0,0799\end{array}$ \\
\hline
\end{tabular}

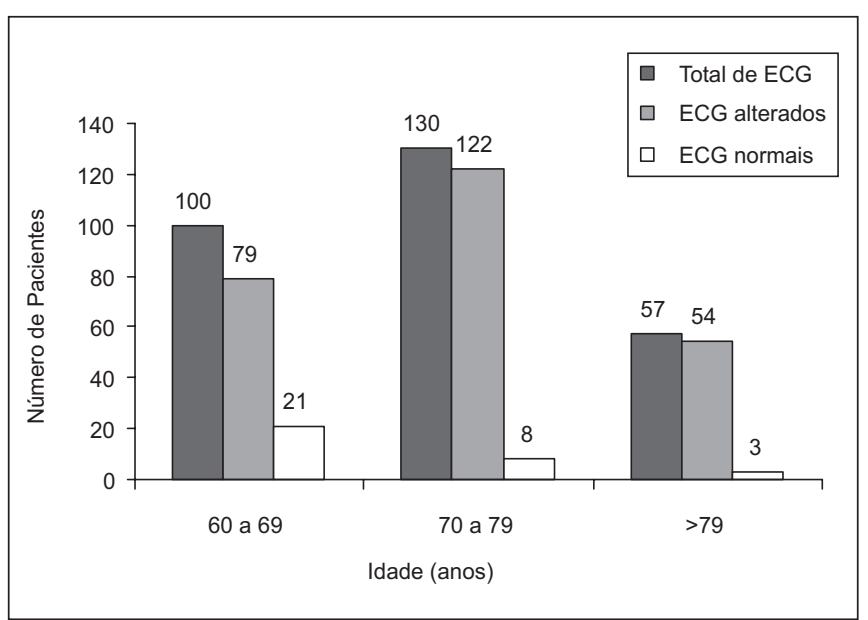

Figura 1 - Distribuição do Total de Pacientes com ECG, de Pacientes com ECG Alterados e Normais, de acordo com a Faixa Etária e Resultado da Análise Estatística

$\mathrm{Na}$ tabela IV podem ser observados os resultados dos ECG alterados, que foram classificados em: alteração da repolarização ventricular; alterações do ritmo cardíaco (extrassistolia supraventricular e ventricular, fibrilação atrial, taquicardia e bradicardia sinusais); alterações secundárias à isquemia (zona inativa, inversão de onda T, zona de isquemia); bloqueios de condução (bloqueio atrioventricular de $1^{\circ} \mathrm{grau}$ e total, bloqueio de ramos direito e esquerdo e bloqueio divisional anterior esquerdo) e sobrecargas das câmaras cardíacas (atriais direita e esquerda e ventriculares direita e esquerda). Encontraram-se 97 ECG com laudos com mais de um tipo de alteração, algumas vezes três ou mais e estes foram analisados conjuntamente.

Na tabela $V$ constam os tipos de alterações eletrocardiográficas encontradas e a incidência de complicações intra e pós-operatórias. Dez $(66,6 \%)$ dos pacientes com alterações secundárias à isquemia e $24(36,4 \%)$ daqueles com alteração da repolarização ventricular apresentaram $\mathrm{ClO}$. 
Tabela IV - Tipos de Alterações Eletrocardiográficas (Número e Porcentagem)

\begin{tabular}{|c|c|c|}
\hline & Número Total & $\%$ \\
\hline Alteração da repolarização ventricular & 66 & 5,9 \\
\hline Alterações do ritmo cardíaco & 24 & 9,4 \\
\hline Extrassistolia supraventricular & 5 & \\
\hline Extrassistolia ventricular & 3 & \\
\hline Fibrilação atrial & 6 & \\
\hline Taquicardia sinusal & 1 & \\
\hline Bradicardia sinusal & 9 & \\
\hline Alterações secundárias à isquemia & 15 & 5,9 \\
\hline Bloqueios de condução & 17 & 6,7 \\
\hline Bloqueio átrio-ventricular $1^{\circ} \mathrm{grau}$ & 3 & \\
\hline Bloqueio átrio-ventricular total & 1 & \\
\hline Bloqueio de ramo direito & 6 & \\
\hline Bloqueio de ramo esquerdo & 1 & \\
\hline Bloqueio divisional ântero-superior esquerdo & 6 & \\
\hline Sobrecargas das câmaras cardíacas & 36 & 14,1 \\
\hline Sobrecarga de átrio direito & 2 & \\
\hline Sobrecarga de átrio esquerdo & 5 & \\
\hline Sobrecarga de ventrículo direito & 1 & \\
\hline Sobrecarga de ventrículo esquerdo & 28 & \\
\hline Mais de uma alteração eletrocardiográfica & 97 & 38 \\
\hline Total & 255 & 100 \\
\hline
\end{tabular}

Tabela V - Distribuição dos Pacientes em Relação aos Tipos de Alterações Eletrocardiográficas e Complicações Intra e Pós-Operatórias

\begin{tabular}{lccc}
\hline & $\begin{array}{c}\text { Número de } \\
\text { Pacientes }\end{array}$ & ClO & CPO \\
\hline Alterações da repolarização ventricular & 66 & 24 & 8 \\
Sobrecargas das câmaras cardíacas & 36 & 8 & 5 \\
Bloqueios de condução & 17 & 4 & 3 \\
Alterações secundárias à isquemia (ZI) & $15(2)$ & $10(2)$ & $3(1)$ \\
Alterações do ritmo cardíaco & 24 & 3 & 3 \\
Mais de uma alteração & 97 & 33 & 6 \\
\hline Total & 255 & 82 & 28 \\
\hline
\end{tabular}

$\mathrm{ZI}=$ zona inativa; $\mathrm{ClO}=$ complicações intra-operatórias; $\mathrm{CPO}=$ complicações pós-operatórias

As idades médias e os desvios-padrão dos pacientes sem DCV com ECG normal e alterado foram similares e respectivamente, $74 \pm 7,2$ e 73,6 \pm 7,8 anos.

Na figura 2 pode-se observar a distribuição por faixa etária do número de pacientes sem DCV com ECG realizados, normais e alterados. Não foi possível realizar tratamento estatístico devido ao número pequeno de pacientes com ECG normais em determinadas faixas etárias.

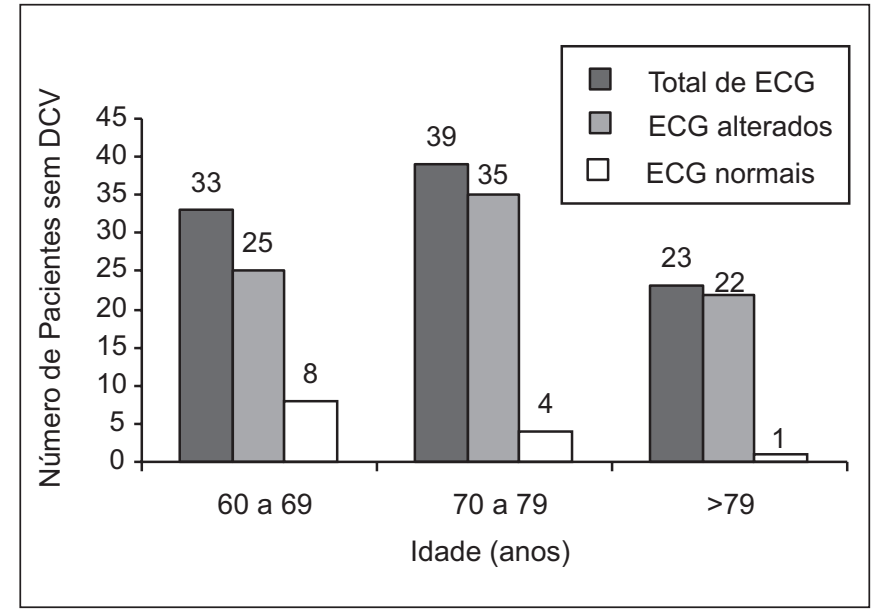

Figura 2 - Distribuição do Total de Pacientes sem DCV com ECG e do Total com ECG Alterados e Normais, de acordo com a Faixa Etária

A tabela VI apresenta a distribuição por faixa etária do número total de pacientes sem DCV com ECG alterados, com e sem $\mathrm{ClO}$ e CPO. Na análise estatística por faixa etária, houve aumento significativo das $\mathrm{CIO}$ com o aumento da idade $\left(\chi^{2}\right.$; $p=0,0283$ ). Devido ao número pequeno de complicações pós-operatórias nos pacientes sem DCV e ECG alterados, não foi realizado tratamento estatístico, mas na análise descritiva não se observou variação uniforme da incidência de CPO com a idade.

Tabela VI - Distribuição por Faixa Etária do Número Total de Pacientes sem DCV: com ECG Alterados e do Número de Pacientes com/sem CIO e CPO e Resultado da Análise Estatística $\left(\chi^{2}\right)$

\begin{tabular}{lccc}
\hline Faixa Etária (anos) & Total & Com/Sem ClO & Com/Sem CPO \\
\hline 60 a 69 & 25 & $3 / 22$ & $1 / 24$ \\
70 a 79 & 35 & $13 / 22$ & $5 / 30$ \\
$>79$ & 22 & $9 / 13$ & $2 / 20$ \\
\hline Total & 82 & $25 / 57$ & $8 / 74$ \\
p & & 0,0283 &
\end{tabular}

$\chi^{2}=$ teste do Qui-quadrado; $\mathrm{CIO}=$ complicações intra-operatórias $\mathrm{CPO}=$ complicações pós-operatórias

Em relação aos tipos de alterações eletrocardiográficas encontradas nos pacientes sem DCV e a incidência de complicações intra e pós-operatórias, verificou-se que 3 (60\%) dos pacientes com alterações secundárias à isquemia e 13 $(38,2 \%)$ daqueles com mais de uma alteração apresentaram CIO (Tabela VII).

As idades médias e desvios-padrão dos pacientes ASA I com ECG normal e alterado foram similares e respectivamente $73,3 \pm 6,5$ e $73,4 \pm 6,8$ anos.

A figura 3 apresenta a distribuição por faixa etária do número total de pacientes ASA I com ECG realizados, normais e alterados. Não foi possível realizar tratamento estatístico devido ao número pequeno de pacientes com ECG normais em determinadas faixas etárias, mas observou-se na análise descritiva elevação do número de ECG alterados coma idade. Vol. 55, № 1, Janeiro - Fevereiro, 2005 
Tabela VII - Distribuição dos Pacientes sem DCV em Relação aos Tipos de Alterações Eletrocardiográficas e Complicações Intra e Pós-Operatórias

\begin{tabular}{lccc}
\hline & $\begin{array}{c}\text { Número de } \\
\text { Pacientes }\end{array}$ & ClO & CPO \\
\hline Alterações da repolarização ventricular & 23 & 6 & 2 \\
Sobrecargas das câmaras cardíacas & 6 & 1 & 0 \\
Bloqueios de condução & 6 & 1 & 0 \\
Alterações secundárias à isquemia & 5 & 3 & 1 \\
Alterações do ritmo cardíaco & 8 & 1 & 1 \\
Mais de uma alteração & 34 & 13 & 4 \\
\hline Total & 82 & 25 & 8 \\
\hline
\end{tabular}

$\mathrm{CIO}=$ complicações intra-operatórias; $\mathrm{CPO}=$ complicações pós-operatórias

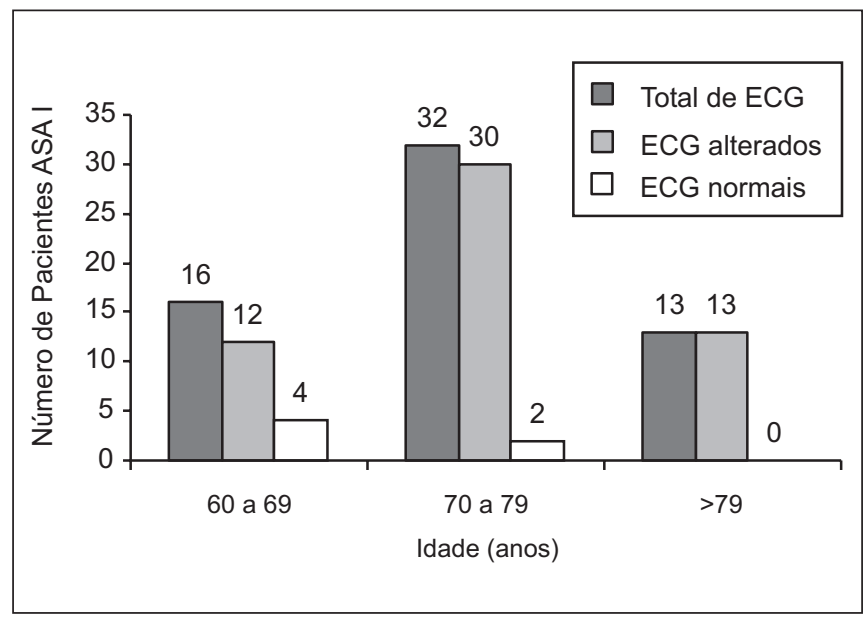

Figura 3 - Distribuição do Total de Pacientes ASA I com ECG e do Total com ECG Alterados e Normais, de acordo com a Faixa Etária

Na tabela VIII encontra-se a distribuição dos pacientes ASAI com ECG normal e alterado em relação às complicações hemodinâmicas intra e pós-operatórias. Devido ao número pequeno de complicações intra e pós-operatórias nos pacientes ASA I com ECG alterados, não foi realizada análise estatística, mas na análise descritiva verificou-se aumento da incidência de $\mathrm{ClO}$ de $16,6 \%$ para $46,1 \%$ com o aumento da idade, o que não aconteceu com a incidência de CPO.

Tabela VIII - Distribuição por Faixa Etária do Número Total de Pacientes ASA I com ECG Alterados e do Número de Pacientes com/sem CIO e CPO

\begin{tabular}{lccc}
\hline Faixa Etária (anos) & Total & Com/Sem ClO & Com/Sem CPO \\
\hline 60 a 69 & 12 & $2 / 10$ & $0 / 12$ \\
70 a 79 & 30 & $9 / 21$ & $1 / 29$ \\
$>79$ & 13 & $6 / 7$ & $2 / 11$ \\
\hline Total & 55 & $17 / 38$ & $3 / 52$ \\
\hline
\end{tabular}

$\mathrm{CIO}=$ complicações intra-operatórias; $\mathrm{CPO}=$ complicações pós-operatórias

Revista Brasileira de Anestesiologia

Vol. 55, No 1, Janeiro - Fevereiro, 2005
No estudo dos tipos de alterações eletrocardiográficas encontradas nos pacientes ASA I e incidência de complicações intra e pós-operatórias, observou-se que, dos pacientes com alterações secundárias à isquemia, $2(100 \%)$ apresentaram CIO e $1(50 \%)$ CPO (Tabela IX).

Tabela IX - Distribuição dos Pacientes ASA I em Relação aos Tipos de Alterações Eletrocardiográficas e Complicações Intra e Pós-Operatórias

\begin{tabular}{lccc}
\hline & $\begin{array}{c}\text { Número de } \\
\text { Pacientes }\end{array}$ & ClO & CPO \\
\hline Alterações da repolarização ventricular & 16 & 7 & 1 \\
Sobrecargas das câmaras cardíacas & 8 & 2 & 0 \\
Bloqueios de condução & 9 & 2 & 0 \\
Alterações secundárias à isquemia & 2 & 2 & 1 \\
Alterações do ritmo cardíaco & 4 & 1 & 0 \\
Mais de uma alteração & 16 & 3 & 1 \\
\hline Total & 55 & 17 & 3 \\
\hline
\end{tabular}

$\mathrm{CIO}=$ complicações intra-operatórias; $\mathrm{CPO}=$ complicações pós-operatórias

Resumindo-se os resultados apresentados, pode-se observar nos três grupos estudados (total de pacientes, pacientes sem DCV e pacientes ASA I), na figura 4 , a incidência por faixa etária de ECG alterados e, nas figuras 5 e 6 nestes mesmos grupos, a incidência de CIO hemodinâmicas nos pacientes com ECG alterados e nos pacientes com alterações secundárias à isquemia, alterações da repolarização ventricular e mais de uma alteração eletrocardiográfica.

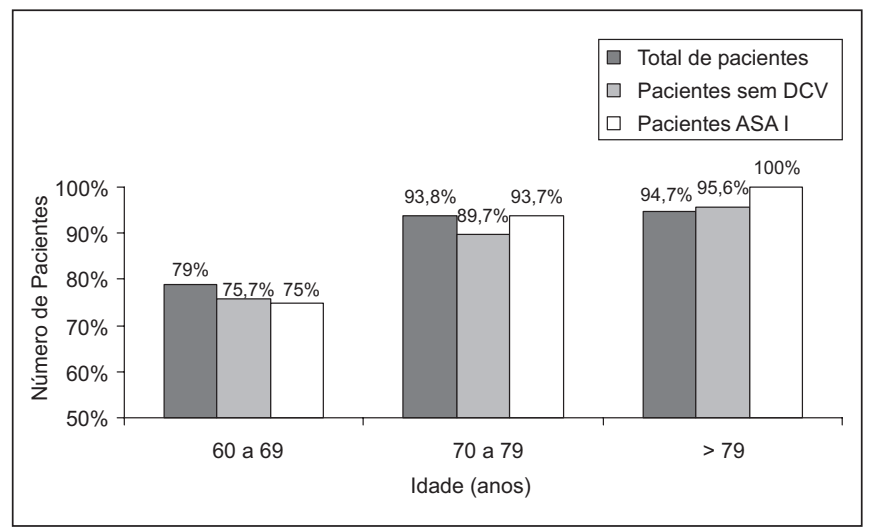

Figura 4 - Distribuição de acordo com a Faixa Etária, da Porcentagem de ECG Alterados no Grupo Total de Pacientes, nos Pacientes sem DCV e ASA I

Total de pacientes do estudo $-\mathrm{n}=287$; pacientes sem DCV $=$ pacientes sem doença cardiovascular $-n=95$; pacientes ASA I $=$ pacientes estado físico ASA I - $n=61$ 


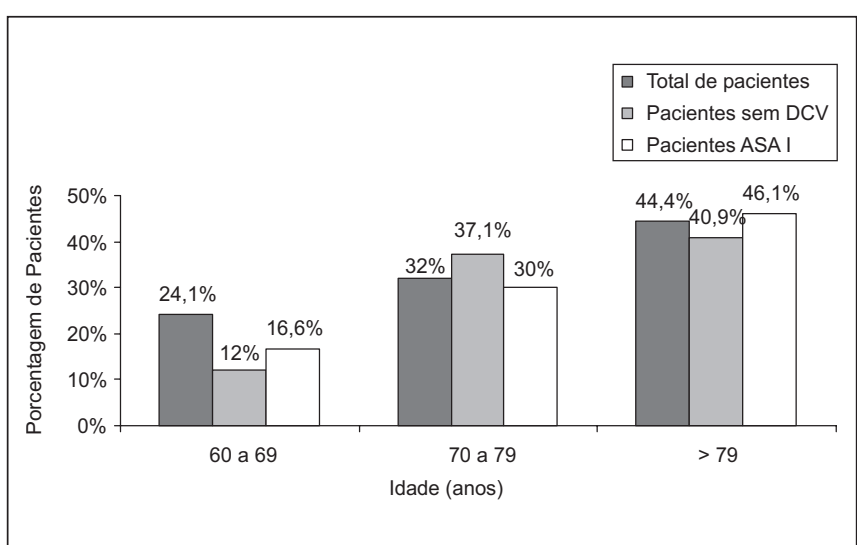

Figura 5 - Distribuição de acordo com a Faixa Etária, da Porcentagem de ECG Álterados com CIO Cardiovasculares no Grupo Total de Pacientes, nos Pacientes sem DCV e ASA I

Total de pacientes do estudo $-n=287$; pacientes sem DCV $=$ pacientes sem doença cardiovascular $-n=95$; pacientes ASA I = pacientes estado físico ASA I - $\mathrm{n}=61$

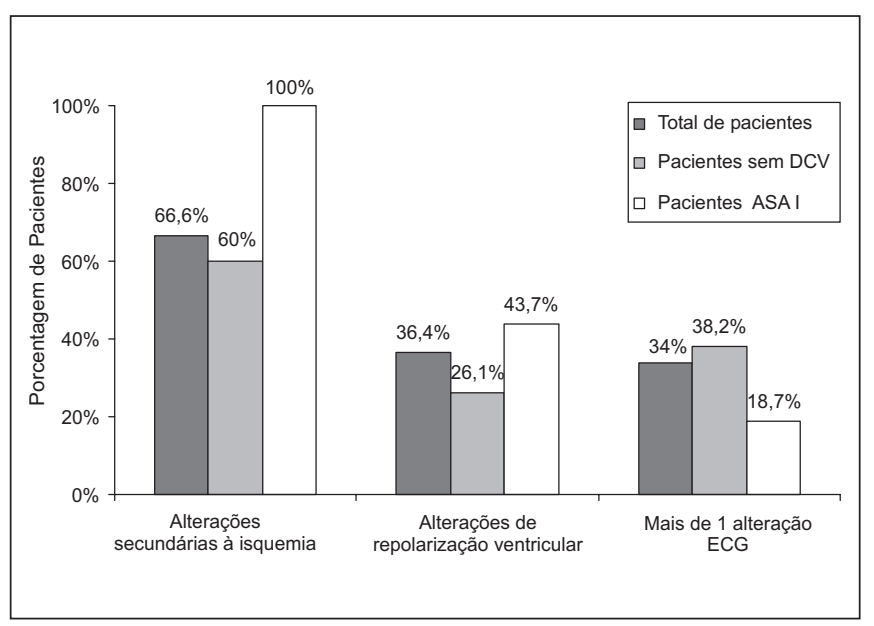

Figura 6 - Distribuição da Porcentagem de Pacientes com Alterações Eletrocardiográficas com ClO Cardiovasculares no Grupo Total de Pacientes, nos Pacientes sem DCV e ASA I Total de pacientes do estudo $-\mathrm{n}=287$; pacientes sem DCV $=$ pacientes sem doença cardiovascular $-\mathrm{n}=95$; pacientes ASA I = pacientes estado físico ASA I - $n=61$

\section{DISCUSSÃO}

O presente estudo, por tratar-se de um estudo de coorte histórica ou coorte retrospectiva, teve algumas limitações, uma vez que dados de prontuários, atestados e formulários preenchidos não obedecem a critérios uniformes ${ }^{17}$.

$\mathrm{Na}$ análise do total de prontuários com ECG verificou-se que $88,85 \%$ apresentavam laudos com alterações. Seymour (1993) ${ }^{18}$ encontrou incidência semelhante de ECG com anormalidades ( $79 \%$ ) num grupo de 222 pacientes cirúrgicos com idade maior que 65 anos.
Amaioria dos pacientes deste estudo era estado físico ASAII e apresentava DCV sintomática, resultados referidos também pela literatura ${ }^{10,19}$.

Não foi encontrada diferença significante entre os grupos com ECG normal e alterado em relação ao estado físico e a presença de DCV, diferentemente de outros autores 8,9,11,19-21

Ao contrário do que se poderia imaginar, que os pacientes sem DCV e/ou os pacientes ASAI fossem os mais "jovens" do grupo de idosos estudados, a idade média destes pacientes foi similar à do grupo como um todo. Dentro destes grupos, os pacientes com ECG normal e aqueles com ECG alterado, também apresentaram idade média similar. Entretanto, na distribuição por faixa etária, observou-se aumento da incidência de alterações eletrocardiográficas com o avanço da idade, em todos os grupos estudados, concordando com a literatura $^{9-11}$. Vale ressaltar que a menor incidência de ECG alterados foi $75 \%$ nos pacientes ASA I, de 60 a 69 anos, chegando a $100 \%$ neste mesmo grupo na faixa etária acima de 79 anos.

As $\mathrm{ClO}$ hemodinâmicas dos pacientes com ECG alterados no grupo total de pacientes, dos pacientes sem DCV e dos pacientes ASA I foi crescente com o avanço da idade, fato não observado com as CPO. Isso sugere uma pergunta: será que os pacientes com ECG alterado sem doença cardiovascular e ASA I têm aumento das CIO com a idade, semelhante ao grupo total de pacientes (no qual estariam incluídos pacientes com doença cardiovascular), devido às alterações fisiológicas relacionadas ao envelhecimento, independentemente da presença de queixas, sinais e sintomas? Baseado nessa hipótese, não estaria então indicada a realização do eletrocardiograma de rotina em todos os pacientes idosos? Em todos os grupos, os pacientes com laudos com alterações secundárias à isquemia, foram os que apresentaram maior incidência de complicações intra-operatórias, o que condiz com a literatura ${ }^{22-26}$. Um dado importante é que, nos pacientes sem DCV e ASA I, estas alterações não estavam acompanhadas de quadro clínico e a incidência de $\mathrm{ClO}$ foi de 60 e $100 \%$, o que mais uma vez sugere a realização do ECG de rotina.

Na literatura nacional, Nascimento e col. (1998) ${ }^{10}$ realizaram estudo prospectivo com o objetivo de avaliar a presença e a distribuição de alterações eletrocardiográficas pré-operatórias, em pacientes ASA I e II sem DCV, ou fator de risco para DCV, divididos por faixa etária, desde 30 anos a $\geq 70$ anos. Consideraram questionável a realização do ECG pré-operatório de rotina em pacientes sem $\mathrm{DCV}$, mas recomendaram que o bom senso e a experiência individual prevalecesse na decisão final.

Na literatura mundial, duas publicações merecem destaque, por terem resumido as evidências disponíveis sobre o ECG pré-operatório: a revisão de Munro e col. (1997) ${ }^{27}$ e a da Força Tarefa $(\mathrm{FT})$ da $\mathrm{ASA}^{28}$, que se ativeram às evidências científicas disponíveis de boa qualidade sobre o valor dos exames pré-operatórios de rotina em pacientes adultos de 1966 a 1996 e até 1999 respectivamente. $A F T^{28}$ da ASA, além disso, coletou a opinião de especialistas, com o objetivo de che- 
gar a um consenso e produzir um guia prático consultivo em avaliação pré-anestésica.

Em relação ao ECG pré-operatório, identificaram 28 e 30 estudos, respectivamente, a maioria deles retrospectivos, dos quais, segundo os autores, apenas dois restringiam-se a pacientes idosos ${ }^{27}$. A prevalência de alterações eletrocardiográficas aumentou exponencialmente com a idade, com a piora do estado físico e com fatores de risco cardíaco. Não houve consenso sobre a idade mínima para realização de rotina do ECG pré-operatório, especialmente nos pacientes sem fatores de risco específicos.

Munro e col. (1997) ${ }^{27}$ concluíram que permanecia a dúvida do benefício da utilização do ECG de rotina, numa população de pacientes assintomáticos, de maior risco de complicações intra-operatórias, como os pacientes idosos. Segundo os autores, embora existissem evidências de boa qualidade de que a incidência de anormalidades dos exames aumentava com a idade, a questão principal seria se a porcentagem de anormalidades não esperadas, que implicava em mudança de conduta, aumentaria com a idade e não existiam evidências disponíveis suficientes para responder essa pergunta.

$A F T^{28}$ da ASA concluiu que a idade podia não ser indicador único para realização de ECG, mas também concluiu que a literatura científica disponível não continha informações suficientemente rigorosas sobre a avaliação pré-anestésica que permitisse recomendações que não fossem ambíguas.

Este estudo corrobora as proposições de diversos estudos, mas principalmente de Munro e col. (1997) ${ }^{27}$ e da FT ${ }^{28}$ da ASA, de que não é possível concluir que se deve realizar ECG de rotina em todos os pacientes idosos, sequer definir um limite mínimo de idade para tanto. No entanto, este estudo mostrou que clinicamente pode ser relevante obter o ECG pré-operatório para os pacientes idosos, até que ensaios clínicos aleatórios sejam realizados.

Assim, dentro das limitações deste estudo retrospectivo, concluímos que para a população cirúrgica idosa da ISCMSP, é válida a rotina de realização do eletrocardiograma como parte da avaliação pré-operatória.

\section{The Validity of the Electrocardiogram Accomplishment in the Elderly Surgical Patient Preoperative Evaluation}

Flávia Salles de Souza, M.D.; José Ricardo Pinotti Pedro, M.D.; Joaquim Edson Vieira, TSA, M.D.; Arthur Vitor Rosenti Segurado, M.D.; Marcos Paulo Ferreira Botelho, M.D.; Lígia Andrade da Silva Telles Mathias, TSA, M.D.

\section{INTRODUCTION}

Life expectancy has increased in the last 100 years and medical progresses seem to be the major contributing factor. More than half of elderly people will be submitted to at least one surgical procedure during life and anesthesiologists have to be prepared to evaluate and understand this increasing number of patients ${ }^{1}$.

For the cardiovascular system, aging is related to a wide variety of structural and functional changes in heart and vessels. In addition to age-related physiological changes, there is increased prevalence of underlying cardiovascular diseases (systemic hypertension, atherosclerosis, acute myocardial infarction, congestive heart failure) which limit even further the functioning of this system ${ }^{2,3}$

Major preoperative and preanesthetic evaluation objective is always to decrease perioperative morbidity-mortality, regardless of patients' age. In this sense, lab tests are being currently indicated as priority according to history and/or physical evaluation findings ${ }^{4}$.

Preoperative ECG is routinely indicated in the attempt to identify cardiovascular diseases and decrease intraoperative morbidity-mortality, however, its influence on pre and intraoperative approaches to prevent cardiovascular complications is controversial ${ }^{5-7}$.

Several authors have described increased ECG changes with age ${ }^{8-11}$.

It is known that silent myocardial ischemia (SMI) is more frequent in the elderly ${ }^{12}$ and that recent infarction is a strong predictor for increased perioperative cardiovascular risk ${ }^{13}$.

However, there are still divergences in the literature about the accuracy of preoperative ECG in predicting perioperative cardiovascular complications in elderly patients and, as a consequence, about its routine indication for this group of patients ${ }^{10,12,14-16}$.

This study aimed at evaluating the usefulness of routine preoperative ECG in a population of elderly surgical patients.

\section{METHODS}

After the approval of the Research Ethics Committee, Hospital Central, Irmandade da Santa Casa de Misericórdia, São Paulo, this retrospective study consisted of the evaluation of elderly patients records (60 years or beyond) in the period April 1 to September 30, 2000. Patients were submitted to different surgical procedures under anesthesia, with preoperative ECG performed at admission or in outpatient regimen until 6 months before admission, with report of a cardiologist or clinician working for the institution.

Data collected were: gender, age, physical status (ASA), presence of signs or symptoms of cardiovascular disease or other related diseases, electrocardiogram, type of surgery and anesthetic technique, intraoperative hemodynamic complications not related to surgical adverse effects (heart rate or blood pressure increase/decrease above $20 \%$ of baseline and arrhythmias), and postoperative complications.

Data were evaluated in the following sequence:

1. Incidence of ECG performed in the conditions established by this study; 
2. Incidence of normal and abnormal ECG with regard to variables: age, age bracket (60-69, $70-79$ and $\geq 80$ years of age), physical status, presence of cardiovascular disease, intra and postoperative complications. Incidence of abnormal ECG associated to intra and postoperative complications according to age distribution, types of electrocardiography changes and incidence of intra and postoperative complications related to observed abnormalities;

3. Evaluation of patients with no cardiovascular disease and ASA I to: check the incidence of abnormal ECG according to age distribution and the incidence of associated intra and postoperative complications; check the types of electrocardiography changes and the incidence of intra and postoperative complications related to observed changes.

Results were submitted to descriptive analysis. Non-paired Student's $t$ test was used to compare age results. Chi-square test $\left(\chi^{2}\right)$ was used to compare results of remaining variables. $\mathrm{P}<0.05$ was considered statistically significant. Tests used are part of the statistical suite Sigma Stat for Windows, version 2.03, SPSS Inc.

\section{RESULTS}

From 481 evaluated files, 194 were excluded for being patients submitted to surgery without ECG or with ECG not complying with our protocol. Total number of patients included was 287 , of whom 255 had abnormal ECG (88.85\%).

Mean age (standard deviations) of patients with normal and abnormal ECG were respectively 74.2 (7.1) and 73.5 (8.2) years without statistical difference among groups (non-paired $t$ test - $p=0.643$ ). There were also no significant differences in gender distribution with majority of males in both groups $\left(\chi^{2}-p=0.544\right)$.

There were no significant differences among groups in normal and abnormal ECG and physical status (ASA) $\left(\chi^{2}\right)$, with most patients with abnormal ECG, regardless of physical status (Table I).

Table I - Distribution of Patients with Normal and Abnormal ECG related to Physical Status Classification and Statistical Analysis Result $\left(\chi^{2}\right)$

\begin{tabular}{lccc}
\hline Physical Status & Normal ECG & Abnormal ECG & Total \\
\hline ASA I & $6(9.8 \%)$ & $55(90.2 \%)$ & 61 \\
ASA II & $22(11.9 \%)$ & $163(88.1 \%)$ & 185 \\
ASA III & $4(10.5 \%)$ & $34(89.5 \%)$ & 38 \\
ASA IV & $0(0 \%)$ & $3(100 \%)$ & 3 \\
\hline Total & 32 & 255 & 287 \\
\hline
\end{tabular}

$\chi^{2}=$ Chi-square test $-p=0.722$

When normal and abnormal ECG patients were compared for the presence of symptomatic cardiovascular disease (SCD) and intraoperative (IOC) and postoperative (POC) complica- tions there were no statistically significant differences among groups, that is, the number of patients with or without SCD; with or without IOC, with or without POC was similar in both normal and abnormal ECG groups (Table II).

Table II - Distribution of Patients with Normal and Abnormal ECG related to the Presence of Cardiovascular Disease, Intra and Postoperative Complications and Statistical Analysis Result $\left(\chi^{2}\right)$

\begin{tabular}{lcccc}
\hline & & $\begin{array}{c}\text { Normal } \\
\text { ECG } \mathrm{n}(\%)\end{array}$ & $\begin{array}{c}\text { Abnormal } \\
\text { ECG } \mathrm{n}(\%)\end{array}$ & $\mathrm{p}\left(\chi^{2}\right)$ \\
\hline Cardiovascular & yes & 19 & 173 & 0.255 \\
disease & $(\mathrm{n}=192)$ & $(59.4)$ & $(67.8)$ & \\
& $\mathrm{no}$ & 13 & 82 & \\
Intraoperative & yes & $(40.6)$ & $(32.2)$ & \\
complications & $(\mathrm{n}=89)$ & $(21.9)$ & $(32.1)$ & 0.155 \\
& no & 25 & 173 & \\
& $(\mathrm{n}=198)$ & $(78.1)$ & $(67.9)$ & \\
Postoperative & yes & 1 & 28 & 0.338 \\
complications & $(\mathrm{n}=29)$ & $(3.1)$ & $(11)$ & \\
& no & 31 & 227 & \\
& $(\mathrm{n}=258)$ & $(96.9)$ & $(89)$ & \\
\hline
\end{tabular}

Figure 1 shows age bracket distribution and total number of patients with normal and abnormal ECG. There have been statistical differences in age bracket, and in the number of normal and abnormal ECG $\left(\chi^{2} ; p=0.0006\right)$.

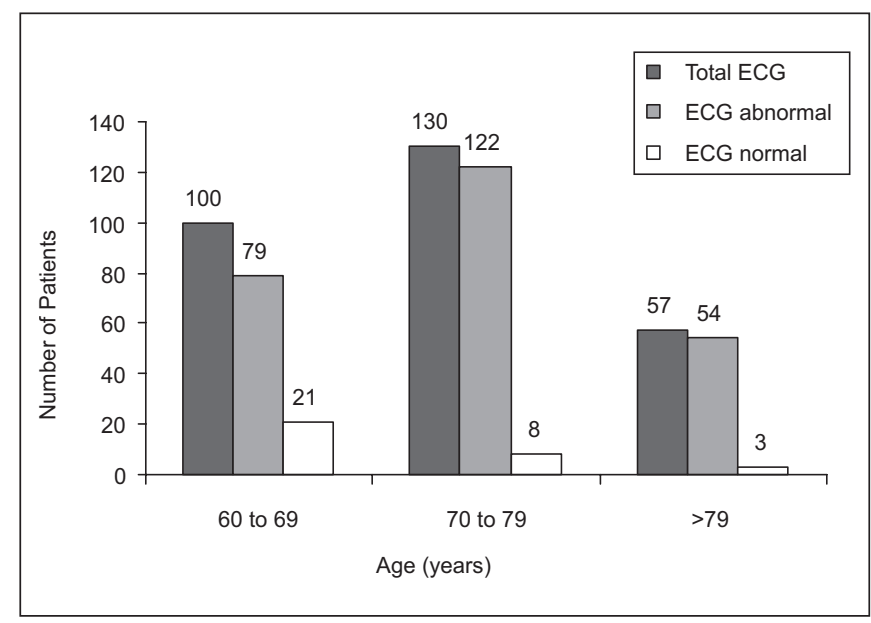

Figure 1 - Distribution of Total Patients with ECG, of Patients with Abnormal and Normal ECG, according to Age Bracket and Statistical Analysis Result

Table III shows results of age distribution of the total number of patients with abnormal ECG with or without IOC and POC and statistical analysis result $\left(\chi^{2}\right)$. There has been significant increase in IOC with increased age $(p=0.0146)$, what was not seen with POC $(p=0.0799)$. 
Table III - Distribution by Age Bracket of Total Number of Patients with Abnormal ECG and Number of Patients with Abnormal ECG with / without IOC and OPC and Statistical Analysis Result $\left(\chi^{2}\right)$

\begin{tabular}{lccc}
\hline Age Bracket & & \multicolumn{2}{c}{ Total Patients } \\
\hline (years) & Total & $\begin{array}{c}\text { With/Withour } \\
\text { IOC }\end{array}$ & $\begin{array}{c}\text { With/Withour } \\
\text { POC }\end{array}$ \\
\hline 60 to 69 & 79 & $19 / 60$ & $13 / 66$ \\
70 to 79 & 122 & $39 / 83$ & $11 / 111$ \\
$>79$ & 54 & $24 / 30$ & $4 / 50$ \\
\hline Total & 255 & $82 / 173$ & $28 / 227$ \\
p & & 0.0146 & 0.0799 \\
\hline
\end{tabular}

$\chi^{2}=$ Chi-square test; IOC = intraoperative complications; $\mathrm{POC}=$ postoperative complications

Table IV shows abnormal ECG results, which were classified as: ventricular repolarization changes, cardiac rhythm changes (supraventricular and ventricular extra-systole, atrial fibrillation, sinusal tachycardia and bradycardia); changes secondary to ischemia (inactive zone, T wave inversion, ischemic zone); conduction blockades $\left(1^{\text {st }}\right.$ degree and total atrioventricular block, right and left branches block and left anterior divisional block) and cardiac chambers overload (right and left atrial and right and left ventricular chambers). There were 97 ECG with more than one abnormality, sometimes three or even more, and these were jointly evaluated.

Table IV -Types of Electrocardiographic Changes (Number and Percentage)

\begin{tabular}{|c|c|c|}
\hline & Total & $\%$ \\
\hline Ventricular repolarization changes & 66 & 25.9 \\
\hline Cardiac rhythm changes & 24 & 9.4 \\
\hline Supraventricular extrasystole & 5 & \\
\hline Ventricular extrasystole & 3 & \\
\hline Atarial fibrillation & 6 & \\
\hline Sinusal tachycardia & 1 & \\
\hline Sinusal bradycardia & 9 & \\
\hline Changes secondary to ischemia & 15 & 5.9 \\
\hline Conduction blockades & 17 & 6.7 \\
\hline $1^{\text {st }}$ degree atrioventriculat block & 3 & \\
\hline Atrioventricular total block & 1 & \\
\hline Right branch block & 6 & \\
\hline Left branch block & 1 & \\
\hline Left antero-superior divisional block & 6 & \\
\hline Cardiac chambers overloads & 36 & 14.1 \\
\hline Right atrium overload & 2 & \\
\hline Left atrium overload & 5 & \\
\hline Right ventriculum overload & 1 & \\
\hline Left ventriculum overload & 28 & \\
\hline More than one ECG change & 97 & 38 \\
\hline Total & 255 & 100 \\
\hline
\end{tabular}

Table V shows types of electrocardiography changes and the incidence of intra and postoperative complications. Ten $(66.6 \%)$ patients with changes secondary to ischemia, and $24(36.4 \%)$ patients with ventricular repolarization changes had IOC. Mean ages and standard deviations of patients without CVD and with normal and abnormal ECG were similar (74 $\pm 7,2$ and $73,6 \pm 7,8$ years, respectively).

Table V - Distribution of Patients related to Types of Electrocardiographic changes and Intra and Postoperative Complications

\begin{tabular}{lccc}
\hline & $\begin{array}{c}\text { Number of } \\
\text { Patients }\end{array}$ & IOC & POC \\
\hline Ventricular repolarization changes & 66 & 24 & 8 \\
Cardiac chambers overloads & 36 & 8 & 5 \\
Conduction Blockades & 17 & 4 & 3 \\
Changes secondary to ischemia (IZ) & $15(2)$ & $10(2)$ & $3(1)$ \\
Cardiac rhythm changes & 24 & 3 & 3 \\
More than one abnormality & 97 & 33 & 6 \\
\hline Total & 255 & 82 & 28 \\
\hline
\end{tabular}

$\mathrm{IZ}=$ inactive $z o n e ; \mathrm{IOC}=$ intraoperative complications; $\mathrm{POC}=$ postoperative complications

Figure 2 shows age distribution of patients without CVD with normal and abnormal ECG. Statistical analysis was not possible due to the small number of patients with normal ECG in certain age brackets.

Table VI shows age distribution of total number of patients without CVD, with abnormal ECG, with and without IOC and POC. There has been significant IOC increase with increased age $\left(\chi^{2} ; p=0.0283\right)$. Statistical analysis was not possible due to the small number of postoperative complications in patients without CVD and abnormal ECG, but descriptive analysis has not shown uniform variation in the incidence of POC with increased age.

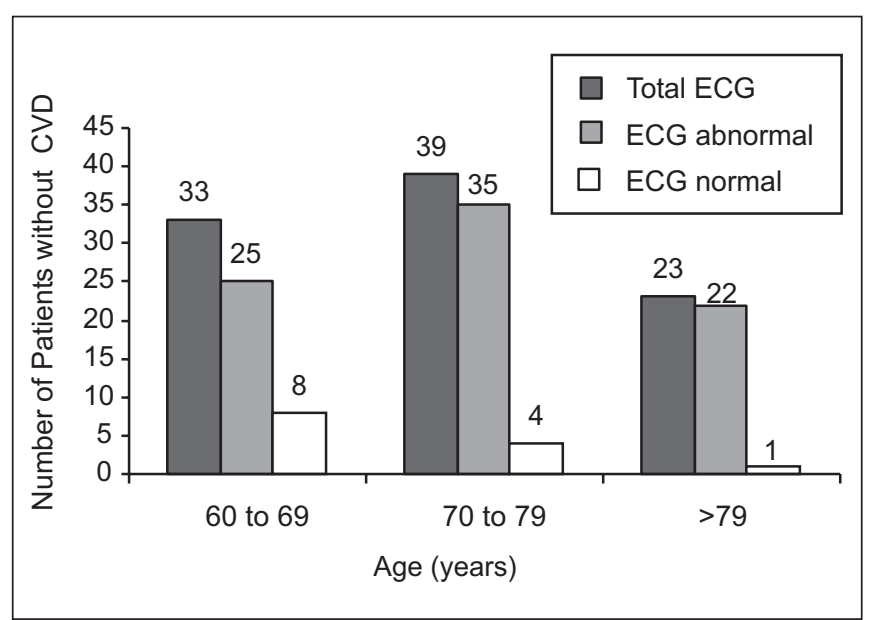

Figure 2 - Distribution of Total Patients without CVD with ECG, and Total of Abnormal and Normal ECG according to Age Bracket 
Table VI - Distribution by Age Bracket of Total Number of CVS-Free Patients; with Abnormal ECG and of Number of Patients with / without IOC and OPC and Statistical Analysis Result $\left(\chi^{2}\right)$

\begin{tabular}{lccc}
\hline Age Bracket (years) & Total & $\begin{array}{c}\text { With/Without } \\
\text { IOC }\end{array}$ & $\begin{array}{c}\text { With/Without } \\
\text { POC }\end{array}$ \\
\hline 60 to 69 & 25 & $3 / 22$ & $1 / 24$ \\
70 to 79 & 35 & $13 / 22$ & $5 / 30$ \\
$>79$ & 22 & $9 / 13$ & $2 / 20$ \\
\hline Total & 82 & $25 / 57$ & $8 / 74$ \\
\hline
\end{tabular}

$\chi^{2}=$ Chi-square test; $I O C=$ intraoperative complications; $P O C=$ postoperative complications

In terms of types of electrocardiography changes in patients without CVD and the incidence of intra and postoperative complications, it has been observed that $3(60 \%)$ patients with changes secondary to ischemia and $13(38.2 \%)$ patients with more than one change have presented IOC (Table VII).

Table VII - Distribution of CVD-Free Patients Related to Types of ECG Abnormalities and Intra and Postoperative Complications

\begin{tabular}{lccc}
\hline & $\begin{array}{c}\text { Number of } \\
\text { Patients }\end{array}$ & IOC & POC \\
\hline Ventricular repolarization changes & 23 & 6 & 2 \\
Cardiac chambers overloads & 6 & 1 & 0 \\
Conduction blockades & 6 & 1 & 0 \\
Changes secondary to ischemia (IZ) & 5 & 3 & 1 \\
Cardiac rhythm changes & 8 & 1 & 1 \\
More than one abnormality & 34 & 13 & 4 \\
\hline Total & 82 & 25 & 8 \\
\hline
\end{tabular}

$\mathrm{IOC}=$ intraoperative complications; $\mathrm{POC}=$ postoperative complications

Mean ages and standard deviations of ASA I patients with normal and abnormal ECG were similar $(73.3 \pm 6,5$ and $73.4 \pm$ 6.8 years, respectively).

Figure 3 shows age distribution of the total number of ASA I patients with normal and abnormal ECG. Statistical analysis was not possible due to the small number of patients with normal ECG in some age brackets, but descriptive analysis has shown an increased number of abnormal ECG with increased age.

Table VIII shows intra and postoperative hemodynamic complications of ASA I patients with normal and abnormal ECG. Statistical analysis was not possible due to the small number of intra and postoperative complications in ASA I patients with abnormal ECG, but descriptive analysis has shown increased incidence of IOC from $16.6 \%$ to $46.1 \%$ with increased age, what was not true for POC.

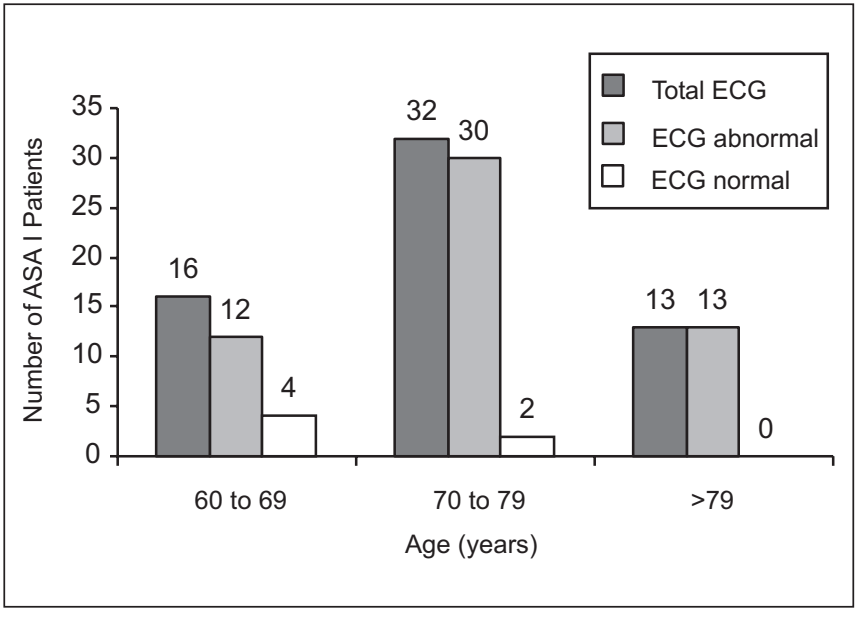

Figure 3 - Distribution of Total ASA I Patients with ECG and of Tota Patients with Abnormal and Normal ECG, according to Age Bracket

Table VIII - Distribution by Age Bracket of Total Number of ASA I Patients with Abnormal ECG and of Number of Patients with / without IOC and OPC

\begin{tabular}{lccc}
\hline Age Bracket (years) & Total & $\begin{array}{c}\text { With/Withour } \\
\text { IOC }\end{array}$ & $\begin{array}{c}\text { With/Without } \\
\text { POC }\end{array}$ \\
\hline 60 to 69 & 12 & $2 / 10$ & $0 / 12$ \\
70 to 79 & 30 & $9 / 21$ & $1 / 29$ \\
$>79$ & 13 & $6 / 7$ & $2 / 11$ \\
\hline Total & 55 & $17 / 38$ & $3 / 52$ \\
\hline
\end{tabular}

$\mathrm{IOC}=$ intraoperative complications; $\mathrm{POC}=$ postoperative complications

In studying types of electrocardiography complications found in ASA I patients and the incidence of changes secondary to ischemia, $2(100 \%)$ had IOC and $1(50 \%)$ had POC (Table IX).

Table IX - Distribution of ASA I Patients Related to Types of ECG Abnormalities and Intra and Postoperative Complications

\begin{tabular}{lccc}
\hline & $\begin{array}{c}\text { Number of } \\
\text { Patients }\end{array}$ & IOC & POC \\
\hline Ventricular repolarization changes & 16 & 7 & 1 \\
Cardiac chambers overloads & 8 & 2 & 0 \\
Conduction blockades & 9 & 2 & 0 \\
Changes secondary to ischemia (IZ) & 2 & 2 & 1 \\
Cardiac rhythm changes & 4 & 1 & 0 \\
More than one abnormality & 16 & 3 & 1 \\
\hline Total & 55 & 17 & 3 \\
\hline
\end{tabular}

$\mathrm{IOC}=$ intraoperative complications; $\mathrm{POC}=$ postoperative complications

In summary, figure 4 shows the incidence of abnormal ECG by age distribution for the three studied groups (total number of patients, patients without CVD and ASA I patients), and figures 5 and 6 show the incidence of hemodynamic IOC in patients with abnormal ECG and patients with changes secondary to ischemia, with ventricular repolarization changes and more than one electrocardiography change. 


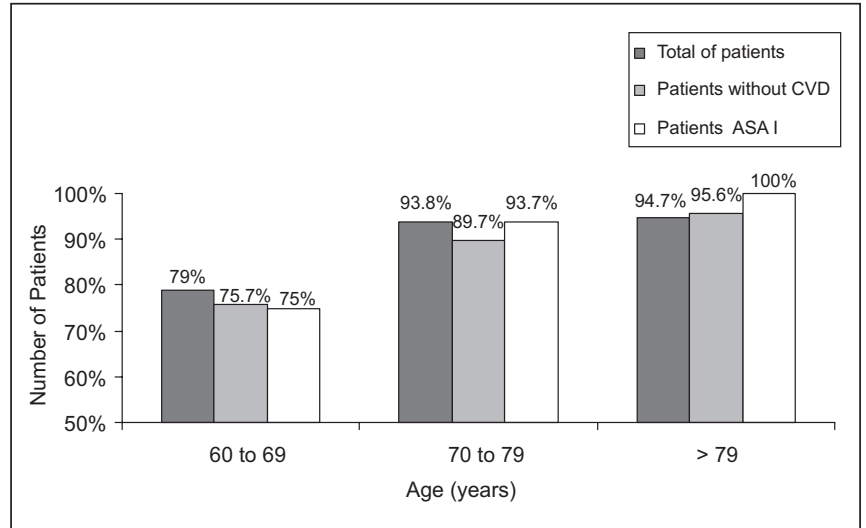

Figure 4 - Distribution of Percentage of Abnormal ECG in Total Group of Patients, in ASA I Patients and Patients without CVD, according to Age Bracket

Total patients of the study $-n=287$; patients without CVD $=$ patients without cardiovascular disease $-n=95$; patients $A S A I=$ physical status ASA I patients - $n=61$

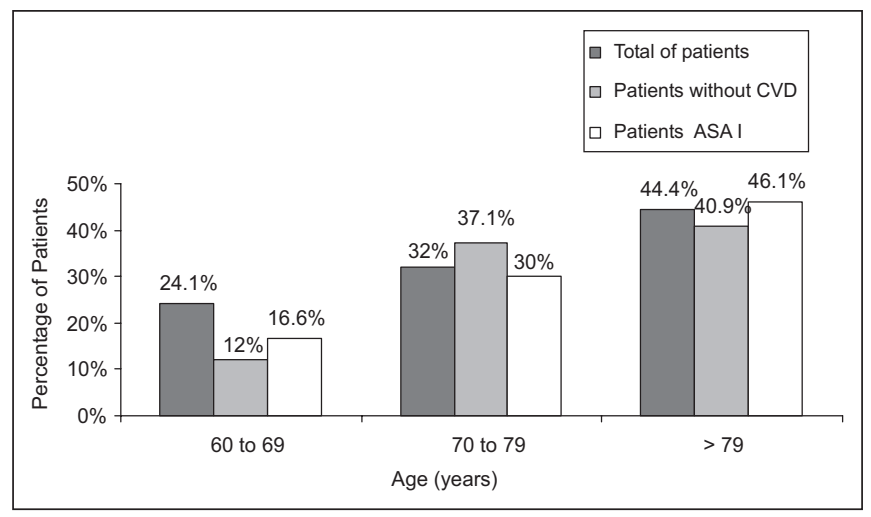

Figure 5 - Distribution of Percentage of Abnormal ECG with Cardiovascular IOC in Total Group of Patients and Patients without CVD and ASA I by Age Bracket

Total patients of the study $-n=287$; patients without CVD $=$ patients without cardiovascular disease $-n=95$; patients ASAI = physical status ASA I patients $-n=61$

\section{DISCUSSION}

For being a historical cohort or retrospective cohort study, our study has suffered some limitations, since records, certificates and forms do not follow uniform criteria ${ }^{17}$.

The analysis of all records with ECG has shown that $88.85 \%$ had abnormal results. Seymour (1993) ${ }^{18}$ has found similar incidence of abnormal ECG $(79 \%)$ in a group of surgical patients aged above 65 years.

Most patients in our study were physical status ASA II with symptomatic CVD, which is in line with the literature ${ }^{10,19}$. We have not found significant differences between groups with normal and abnormal ECG in physical status and presence of CVD, differently from other authors ${ }^{8,9,11,19-21}$.

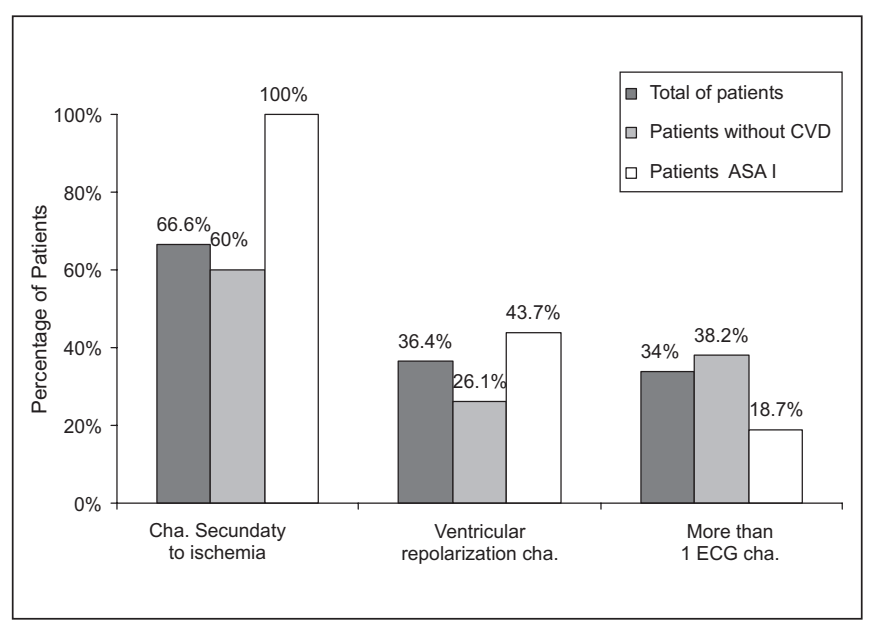

Figure 6 - Distribution of Percentage of Patients with ECG Changes with Cardiovascular IOC in Total Group of Patients, Patients without CVD and ASA I

Total patients of the study $-\mathrm{n}=287$; without CVD $=$ patients without cardiovascular disease $-\mathrm{n}=95$; patients ASA I = physical status ASA I patients $-n=61$; cha. = changes; IOC = intraoperative complications

As opposed to what could be imagined, that is, patients with no CVD and/or ASA I patients would be the "youngest" from the elderly group studied, mean age of these patients was similar to the group as a whole. Within groups, patients with normal or abnormal ECG had also similar mean age. However, there has been increased incidence of electrocardiography abnormalities with increased age in all groups, in agreement with the literature ${ }^{9-11}$. It is worth highlighting that the lowest incidence of abnormal ECG was $75 \%$ in ASA I patients, aged 60 to 69 years and reaching $100 \%$ in the same group but aged above 79 years.

Hemodynamic IOC of patients with abnormal ECG in the total group of patients, in CVD-free patients and in ASA I patients have increased with age, fact not observed with POC. This suggests a question: would patients with abnormal ECG without CVD and ASA I have IOC increase with age similar to the total group of patients (in which cardiovascular patients would be included), due to age-related physiological changes, regardless of complaints, signs and symptoms? Based on this hypothesis, shouldn't routine ECG be indicated to every elderly patient?

In all groups, patients with changes secondary to ischemia were those with the highest incidence of intraoperative complications, which is in line with the literature ${ }^{22-26}$. Important data is that in CVD-free and ASA I patients these changes were not followed by clinical symptoms and the incidence of IOC was $60 \%$ and $100 \%$, once more suggesting routine ECG. In Brazil, Nascimento et al. (1998) ${ }^{10}$ have conducted a prospective study to evaluate the presence and distribution of preoperative ECG changes, in ASA I and II patients with no CVD or CVD risk factor, divided by age bracket from 30 to $\geq 70$ years and have questioned routine preoperative ECG in CVD-free patients, but have recommended that common-sense and individual experience should prevail in the final decision. 
Two publications in the international literature should be stressed because they have summarized available evidences about preoperative ECG: Munro et al. (1997) review 27 and ASA Task Force (TF) review ${ }^{28}$.

Both reviews were limited to high quality scientific evidences on the value of routine preoperative tests for adult patients, in the period 1966 to 1996 and 1966 to 1999 , respectively. In addition, ASA TF ${ }^{28}$ has collected the opinion of specialists aiming at reaching a consensus and develop a practical consulting guide for preanesthetic evaluation.

Authors have identified 28 and 30 studies, respectively, on preoperative ECG, most of them retrospective, of which, according to them, only two were limited to elderly patients ${ }^{27}$. The prevalence of electrocardiography changes has exponentially increased with age, with physical status worsening and cardiac risk factors. There has been no consensus on the lowest age for routine preoperative ECG, especially in patients without specific risks.

Munro et al. (1997) ${ }^{27}$ have concluded that the doubt of the benefit of routine ECG would remain for a population of asymptomatic patients with higher risk of intraoperative complications, such as elderly patients. According to the authors, although there are high quality evidences that the incidence of abnormalities would increase with age, the primary question would be whether the percentage of unexpected abnormalities implying changes in management would increase with age and that there were not enough evidences to answer this question.

ASA TF $^{28}$ has concluded that age could not be the single indicator for ECG, but has also concluded that available scientific literature lacked sufficiently rigorous information on preanesthetic evaluation to allow recommendations that would not be ambiguous.

This study confirms the propositions of different studies, but especially by Munro et al. (1997) ${ }^{27}$ and ASA TF ${ }^{28}$, that it is impossible to conclude that routine ECG should be performed in all elderly patients, not even to define the lowest age for such. However, our study has shown that preoperative ECG might be clinically relevant for elderly patients until randomized clinical trials are conducted.

So, within the limitations of this retrospective study, we have concluded that for ISCMSP's elderly surgical patients, routine ECG as part of preoperative evaluation is a valid approach.

\section{REFERÊNCIAS - REFERENCES}

01. Tonner PH, Kampen J, Scholz J - Pathophysiological changes in the elderly. Best Pract Res Clin Anaesthesiol, 2003;17:163-177.

02. Muravchick S - Physiological changes of aging. ASA Refresher Courses, 2003;31:139-149.

03. Rosenthal RA, Kavic SM - Assessment and management of the geriatric patient. Crit Care Med, 2004;32:(Suppl4):S92-S105.

04. Macpherson DS - Preoperative laboratory testing: should any tests be "routine" before surgery? Med Clin North Am, 1993; $77: 289-308$
05. Goldman L, Caldera DL, Nussbaum SR et al - Multifactorial index of cardiac risk in noncardiac surgical procedures. N Engl J Med, 1977;297:845-850.

06. Mathias LAST, Mathias RS - Avaliação pré-operatória: um fator de qualidade. Rev Bras Anestesiol, 1997;47:335-349.

07. Savaris N, Marcon EM - Avaliação pré-operatória do paciente cardiopata. Rev Bras Anestesiol, 1997;47:350-362.

08. Gold BS, Young ML, Kinman JL et al - The utility of preoperative electrocardiograms in the ambulatory surgical patient. Arch Intern Med, 1992;152:301-305.

09. Escolano F, Gomar C, Alonso J et al - Utilidad del electrocardiograma preoperatorio en cirugía electiva. Rev Esp Anestesiol Reanim, 1996;43:305-309.

10. Nascimento Jr P, Castiglia YMM - O eletrocardiograma como exame pré-operatório do paciente sem doença cardiovascular. É mesmo necessário? Rev Bras Anestesiol, 1998;48:352-361.

11. Garcia-Miguel FJ, Garcia Caballero J, Gomez de Caso-Canto JA - Indicaciones del electrocardiograma para la valoración preoperatorio en cirugía programada. Rev Esp Anestesiol Reanim, 2002;49:5-12.

12. Goldberger AL, O'Konski M - Utility of the routine electrocardiogram before surgery and general hospital admission. Critical review and new guidelines. Ann Int Med, 1986:105:552-557.

13. Eagle KA, Berger PB, Calkins $\mathrm{H}$ et al - ACC/AHA guideline update for perioperative cardiovascular evaluation for noncardiac surgery - Executive summary: a report of the American College of Cardiology/American Heart Association task force on practice guidelines (Committee to update the 1996 guidelines on perioperative cardiovascular evaluation for noncardiac surgery). Anesth Analg, 2002;94:1052-1064.

14. Shah KB, Kleinman BS, Rao TL et al - Angina and other risk factors in patients with cardiac diseases undergoing noncardiac operations. Anesth Analg, 1990;70:240-247.

15. Tait AR, Parr HG, Tremper KK - Evaluation of the efficacy of routine preoperative electrocardiograms. J Cardiothorac Vasc Anesth, 1997;11:752-755.

16. Kiran RP, Delaney CP, Senagore AJ - Preoperative evaluation and risk assessment scoring. Clin Coll R Surg, 2003;16:75-84.

17. Almeida $\mathrm{F}^{\circ} \mathrm{N}$, Rouquayol MZ - Desenhos de Pesquisa em Epidemiologia, em: Introdução à epidemiologia, $3^{\mathrm{a}} \mathrm{Ed}$, Rio de Janeiro, Medsi, 2002;169-214.

18. Seymour DG - The role of the routine pre-operative electrocardiogram in the elderly surgical patient. Age Ageing, 1983;12:97-104.

19. Liu L, Leung J - Predicting adverse postoperative outcomes in patients aged 80 years or older. J Am Geriatr Soc, 2000;48:405-412.

20. Bhuripanyo K, Viwathanatepa M, Prasertchuang C et al - The impact of routine preoperative electrocardiogram in patients age $\geq$ 40 years in Srinagarind Hospital. J Med Assoc Thai, 1992;75:399-406.

21. Velanovich $\mathrm{V}$ - Preoperative screening electrocardiography: predictive value for postoperative cardiac complications. South Med J, 1994;87:431-434.

22. Kannel WB, Abbott RD - Incidence and prognosis of unrecognized myocardial infarction. An update on the Framingham study. N Engl J Med, 1984;311:1144-1147.

23. Tervahauta M, Pekkanen J, Punsar S et al - Resting electrocardiographic abnormalities as predictors of coronary events and total mortality among elderly men. Am J Med, 1996;100:641-645.

24. Landesberg G, Christopherson R, Berlatzky $Y$ et al Perioperative ischemia and cardiac complications in major vascular surgery: importance of the preoperative twelve-lead electrocardiogram. J Vasc Surg, 1997;26:570-578.

Revista Brasileira de Anestesiologia Vol. 55, № 1, Janeiro - Fevereiro, 2005 
25. Suttner SW, Piper SN, Boldt J - The heart in the elderly critically ill patient. Curr Opin Crit Care, 2002;8:389-394.

26. Aronow WS - Prevalence and prognosis in older patients diagnosed by routine electrocardiograms. Geriatrics, 2003;58: 24-40.

27. Munro J, Booth A, Nicholl J - Routine preoperative testing: a systematic review of the evidence. Health Technol Assess, 1997;1:1-62.

28. Pasternak LR, Arens JF, Caplan RA et al - Practice advisory for preanesthesia evaluation - a report by the ASA Task Force on preanesthesia evaluation. Anesthesiology, 2002;96:485-496.

\section{RESUMEN}

Souza FS, Pedro JRP, Vieira JE, Segurado AVR, Botelho MPF, Mathias LAST - Validez de la Rutina de Realización del Electrocardiograma en la Evaluación Pre-Operatoria de Ancianos

JUSTIFICATIVA Y OBJETIVOS: Con los avances médicos y el aumento de la expectativa de vida, la población de ancianos sometidos a procedimientos quirúrgicos viene aumentando. El paciente de edad tiene mayor morbiletalidad cardiaca peri-operatoria que el paciente joven, también cuando asintomático. El electrocardiograma (ECG) ha sido pedido en el período pre-operatorio en la tentativa de reducir la morbiletalidad intra-operatoria, sin embargo su eficacia es controversa. No hay acuerdo en la literatura a respecto de la indicación del ECG en pacientes ancianos en el período pre-operatorio, lo que motivó la realización de este estudio. El objetivo fue verificar retrospectivamente la validez de la rutina de realización del ECG en el período pre-operatorio en una población de pacientes ancianos quirúrgicos.

MÉTODO: Análisis retrospectivo de prontuarios de pacientes a partir de 60 años, sometidos a cirugías diversas, durante el período de 6 meses. Los datos fueron colectados para análisis descriptivo de la población estudiada; evaluación de la incidencia y de los tipos más frecuentes de anormalidad de los ECG; análisis comparativo de los pacientes con ECG normal y alterado con relación a las diversas variables - edad, perfil de edad, estado físico, presencia de enfermedad cardiovascular (DCV), complicaciones intra (CIO) y post-operatorias (CPO). También fueron evaluados los pacientes sin DCV y aquéllos con estado físico ASA I con relación a las CIO y CPO, según los tipos de alteraciones del ECG y edad del paciente.

RESULTADOS: Fueron analizados los prontuarios de 481 pacientes, de los cuales 287 contenían ECG y de éstos, $88,8 \%$ presentaban anormalidades, siendo más frecuente, la alteración de la repolarización ventricular. No fue observada influencia de las variables estudiadas sobre la incidencia de ECG alterados. La incidencia de alteraciones del ECG aumentó con el avance de la edad en todos pacientes estudiados. Con el avance de la edad también ocurrió aumento de la incidencia de pacientes con ECG alterados asociados con complicaciones intra-operatorias. Las anormalidades electrocardiográficas fueron relevantes con relación a la incidencia de complicaciones intra-operatorias en todos los grupos estudiados, principalmente las alteraciones secundarias a la isquemia.

CONCLUSIONES: Este estudio mostró que, para la población quirúrgica anciana estudiada, es válida la rutina de realización del electrocardiograma como parte de la evaluación pre-operatoria. 